\title{
Inquérito sobre a qualidade de vida relacionada à saúde em crianças e adolescentes portadores de artrites idiopáticas juvenis
}

\author{
Health related quality of life survey about children and adolescents \\ with juvenile idiopathic arthritis
}

Tatiana B. Brasil ${ }^{1}$, Virginia P.L. Ferriani ${ }^{2}$, Claudia S.M. Machado ${ }^{3}$

\section{Resumo}

Objetivo: estudar o impacto da artrite crônica na qualidade de vida relacionada à saúde, por meio de dois instrumentos autoaplicáveis: a versão destinada aos pais do Childhood Health Assessment Questionnaire (CHAQ) e do Child Health Questionnaire (CHQ) PF50 ${ }^{\circledR}$.

Método: os dois questionários foram respondidos por 36 pais, após instruções durante 1 a 2 visitas clínicas, sendo os índices da Capacidade Funcional (CHAQ), Físico e Psicossocial (CHQ) comparados com as medidas essenciais de atividade da doença: (1) a avaliação global pelo médico, (2) a avaliação global pelos pais, ambas em escala analógica visual de $10 \mathrm{~cm}$, (3) o número de articulações ativas, (4) o numero de articulações com limitação dos movimentos, (5) a velocidade de hemossedimentação.

Resultados: houve diferença significante entre os grupos oligoarticular e poliarticular, sendo os índices de atividade maiores no poliarticular, com exceção da velocidade de hemossedimentação, avaliação global pelos pais e índice psicossocial, confirmando diferentes níveis de percepção pelos pais sobre a atividade e prognóstico. A melhor responsividade estatística frente à intervenção terapêutica, em duas visitas consecutivas, foi a medida da avaliação global pelo médico entre as subjetivas, tendo as medidas avaliadas por instrumentos de responsividade intermediária, quando comparadas com o numero de articulações ativas, número de articulações com limitação dos movimentos e a velocidade de hemossedimentação.

Conclusão: a medida da responsividade de dois instrumentos de avaliação funcional e da qualidade de vida indicou a sua sensibilidade relativa para estimar a melhora clínica em pacientes com artrites idiopáticas juvenis, recebendo tratamento específico.

J Pediatr (Rio J) 2003;79(1):63-8: artrites idiopáticas juvenis, qualidade de vida relacionada à saúde, avaliação funcional, instrumentos auto-aplicáveis, prognóstico.

\begin{abstract}
Objective: to study the impact of chronic arthritis on health related quality of life by means of two self-reported tools: the parents' version of the Childhood Health Assessment Questionnaire (CHAQ) and the Childhood Health Questionnaire PF50 ${ }^{\circledR}$ (CHQ).
\end{abstract}

Methods: both tools were filled in after proper instructions by 36 parents, during 1-2 clinic visits. The Disability Index (CHAQ) and the Physical and Psychosocial scores (CHQ) were compared to the core set of outcome measures, namely 1) physician's global assessment, 2) parents' global assessment, both scored by $10 \mathrm{~cm}$ visual analogue scale, 3) number of joints with active arthritis, 4) number of joints with limited range of motion, 5) erythrocyte sedimentation rate.

Results: there was significant difference for all measures of disease activity, being higher in the polyarticular as compared to oligoarticular except for erythrocyte sedimentation rate, parents' global assessment, and psychosocial score. This leads to different parents' perceptions of disease activity and outcome. The responsiveness of the outcome measures during two follow-up visits of patients receiving active treatment indicated better responsiveness of physicians' global assessment among the subjective measures, and intermediate responsiveness of the self-reported measures in comparison to the number of active and limited joints, and erythrocyte sedimentation rate.

Conclusions: the responsiveness of two health related quality of life tools indicates their relative sensitivity for assessing clinical improvement during active treatment in Juvenile Idiopathic Arthritis patients.

J Pediatr (Rio J) 2003;79(1):63-8: juvenile idiopathic arthritis, health related quality of life, functional assessment, self-reported tools, outcome.

1. Graduanda em Medicina, Faculdade de Medicina de Botucatu, UNESP.

2. Professora Assistente Doutora, Departamento de Puericultura e Pediatria da Faculdade de Medicina de Ribeirão Preto, USP.

3. Professora Assistente Doutora, Departamento de Pediatria da Faculdade de Medicina de Botucatu, UNESP.

Este trabalho teve o suporte da FAPESP- Fundação de Amparo à Pesquisa do Estado de São Paulo, para a Bolsa de Iniciação Científica de Tatiana B. Brasil. 
A avaliação da atividade inflamatória em artrites crônicas, em adultos e crianças, inclui medidas clínicas, como a mobilidade articular, deformidades articulares, alterações radiológicas, medidas laboratoriais e também as medidas da capacidade funcional. Entretanto, estes são de fato descritores da doença, e refletem a percepção, pelo médico, da condição clínica do paciente, mas não refletem os valores e a percepção do paciente, ou de seus pais, sobre o seu estado de saúde.

$\mathrm{Na}$ última década, grande ênfase tem sido dada para incorporar os valores e a percepção do paciente sobre o seu estado de saúde, e diversos instrumentos têm sido desenvolvidos com esta finalidade ${ }^{1-3}$. A "qualidade de vida" em crianças tem sido definida como um conceito subjetivo e multidimensional, que inclui a capacidade funcional e a interação psicossocial da criança e de sua família, enquanto a "qualidade de vida relacionada ao estado de saúde" incorpora a avaliação dos sintomas físicos, capacidade funcional e o impacto psicossocial da doença sobre a criança e a família ${ }^{4}$. Em duas revisões editoriais recentes ${ }^{5,6}$, os autores enfatizam um grande número de instrumentos em desenvolvimento e a necessidade de adaptação cultural, assim como a adequação destes instrumentos para aplicação aos pais e aos pacientes. Em um artigo original, Feldman e col., 2000 ${ }^{7}$, utilizando-se de um instrumento desenvolvido para mensurar a qualidade de vida e qualidade de vida relacionada ao estado de saúde - quality of my life -, compararam os seus índices com as medidas da capacidade física e funcional, e observaram a variabilidade das medidas face a uma ampla variação etária, de diagnósticos e de capacidade física. É importante ressaltar que estes indicadores devem ser capazes de detectar as alterações clínicas importantes e de demonstrar os efeitos do tratamento, mesmo em pequenas séries de pacientes, pois as doenças reumáticas pediátricas são consideradas raras.

Com o objetivo de estudar o impacto das artrites crônicas nos aspectos físicos, funcionais e psicossociais da qualidade de vida relacionada à saúde em pacientes portadores de artrites idiopáticas juvenis, foram utilizados, no presente estudo, dois instrumentos auto-aplicáveis, a versão destinada aos pais do Childhood Health Assessment Questionnaire - CHAQ e do Child Health Questionnaire CHQ-PF50 ${ }^{\circledR}$ (Parents' form), estimando-se a utilidade destes instrumentos frente aos critérios de melhora ou piora clínica na prática diária, e relacionando os índices da capacidade física e grau de satisfação com os parâmetros clínicos de atividade da doença.

\section{Métodos \\ Casuística}

Durante o período de fevereiro de 2000 a outubro de 2001 , foram recrutados 43 pacientes cujos pais se declararam alfabetizados. Após obtenção do consentimento livre e esclarecido pelos pais ou responsáveis, solicitou-se o preenchimento dos questionários, contendo as informações sobre a pesquisa e instruções para preenchimento dos questionários. Trinta e seis pacientes com visitas regulares ao serviço neste período e cujos pais preencheram voluntariamente os dois questionários, com aproveitamento total de ambos, foram incluídos no estudo. Esta foi uma amostra conveniente, recrutada de uma coorte de 150 pacientes com artrite crônica, registrados no ambulatório de reumatologia pediátrica do Hospital das Clínicas da Faculdade de Medicina de Botucatu, um serviço de referência secundária e terciária em Pediatria.

Foram avaliados 36 casos de artrites idiopáticas juvenis $^{8}, 17$ com evolução oligoarticular e 19 poliarticular, e comparados quanto aos descritores, medidas de atividade, avaliação funcional e da qualidade de vida. Durante o período de estudo, 30 pacientes receberam tratamento farmacológico ativo. Os do grupo oligoarticular receberam antiinflamatórios não-esteroidais e injeções intra-articulares de corticosteróides $(n=14)$. Nos casos com evolução poliarticular, além do uso regular de antiinflamatórios nãoesteroidais e corticosteróides orais $(\mathrm{n}=16), 13$ pacientes iniciaram o uso de metotrexate oral ou parenteral como única droga com ação modificadora de atividade da doença, e foram avaliados em duas visitas com intervalo de 4 a 6 meses.

A atividade da doença foi avaliada e classificada em quatro categorias: (1) ativa, com aumento do número das articulações com sinovite ativa, apesar do tratamento; (2) estável, o número de articulações com sinovite permaneceu estável na vigência de tratamento; (3) inativa, nenhuma evidência de sinovite, e sem medicação por período menor que dois anos; (4) em remissão, nenhuma evidência de sinovite, e sem medicação por período maior que dois anos 9 .

Os parâmetros clínicos e laboratoriais e as medidas de atividade, padronizadas internacionalmente, foram: (1) a avaliação global da atividade pelo médico em escala analógica visual (VAS) de $10 \mathrm{~cm}$, ancorada em dois pontos, "inativa" e "muito grave"; (2) a avaliação global da atividade pelos pais em escala analógica visual de $10 \mathrm{~cm}$ (VAS), ancorada em dois pontos, "muito bem" e "muito mal"; (3) a contagem do número de articulações com sinovite ativa, ou número de articulações ativas; (4) a contagem das articulações com limitação dos movimentos; (5) a velocidade de hemossedimentação como parâmetro laboratorial da atividade inflamatória; e (6) capacidade funcional por meio de instrumento específico ${ }^{10}$.

\section{Avaliação funcional e da qualidade de vida}

A avaliação funcional foi realizada por meio da aplicação do CHAQ - Childhood Health Assessment Questionai$r e$, versão destinada aos pais ${ }^{11}$, adaptada e válida ${ }^{12-14}$, que mensura a capacidade funcional e independência em oito atividades da vida diária, estimando-se o grau de dificuldade ou limitações atribuídas à doença numa escala de 0-3, sendo os maiores valores indicativos de menor capacidade. 
A qualidade de vida relacionada à saúde foi avaliada por meio do Child Health Questionnaire - CHQ - PF50 (Landgraf et al., 1999- Healthact - Boston $\left.{ }^{\circledR}\right)^{15}$, em sua versão destinada aos pais ${ }^{14}$, aplicável para crianças com mais de cinco anos de idade por meio de quinze conceitos em saúde. Cada conceito tem uma pontuação de $0-100$, com a maior pontuação indicando melhor estado de saúde, grau de satisfação e bem-estar, e resultam em dois índices sumários - o físico e o psicossocial (0-70).

Foram analisados apenas os questionários válidos, ou seja, respondidos integralmente. Houve aproveitamento de 90\% dos questionários do $\mathrm{CHAQ}$ e $83,7 \%$ do $\mathrm{CHQ}$.

\section{Análise estatística}

A comparação entre os valores das medidas clínicas, dos índices da capacidade funcional e qualidade de vida relacionada à saúde, entre os grupos oligoarticular e poliarticular, foi pelo teste de Mann Whitnney. A comparação entre as medidas realizadas num mesmo paciente em visitas consecutivas foi pelo teste de Wilcoxon. A responsividade estatística dos índices das medidas clínicas, funcionais e da qualidade de vida foi calculada pela Standardized Response Median (SRM) e pelo Effect Size (ES) ${ }^{17}$. A SRM foi calculada como a variação da mediana da visita inicial para a visita final, dividido por $3 / 4$ da diferença da variação interquartil, entre a primeira e segunda visita. O índice ES é a variação da mediana da visita inicial para a visita final, dividido por 3/4 da mediana do índice da visita inicial. Assim, os maiores valores da SEM e ES indicam maior responsividade. Utilizou-se o software estatístico Statmate - Prism Graph Pad 3.0 ${ }^{\circledR}$.

\section{Resultados}

Trinta e seis pacientes, sendo 14 do sexo masculino, entre 3,3-15,4 (mediana 10,3) anos foram incluídos. A duração da doença na primeira avaliação variou de $0,2-8$ (mediana 2,6) anos. Entre estes, 4 pacientes apresentavam anticorpos antinucleares, e 1 apresentava iridociclite crônica. A descrição clínica sumária da casuística estudada é apresentada na Tabela 1.

A comparação das medidas clínicas de atividade da doença, apresentada na Tabela 2, indica que os grupos oligoarticular e poliarticular não diferiram quanto à idade, à duração da doença e à velocidade de hemossedimentação, notando-se diferença estatisticamente significante no número de articulações com inflamação ativa, número de articulações com limitação dos movimentos e capacidade funcional. Entre as medidas subjetivas, a da atividade da doença avaliada pelo médico, em escala analógica visual, mostrou diferença significante entre os grupos oligoarticular e poliarticular, contudo, estes grupos não diferiram pela medida da percepção pelos pais na avaliação global do bem-estar. Por outro lado, a avaliação subjetiva, pelos pais, da escala de dor indicou diferença significante entre estes grupos, tendo o grupo poliarticular maiores índices de dor, mostrando assim diferentes níveis de percepção pelos pais sobre a atividade.

Houve diferença estatística entre os índices do CHAQ dos grupos oligoarticular e poliarticular, tendo o grupo poliarticular maior limitação ou incapacidade.Verificou-se que os índices de cada uma das oito categorias do CHAQ foram mais elevados no grupo poliarticular em relação ao grupo oligoarticular. Os relatos pelos pais sobre a necessidade de adaptações e aparelhos foram registrados em apenas três questionários, na categoria apanhar (adaptador de lápis, abridor de latas), mas o auxílio de outra pessoa para a realização de tarefas foi registrado em 15 dos questionários, nas categorias vestir-se, levantar-se, andar, higienizarse, alcançar, apanhar e realizar atividades e tarefas domésticas, sendo o item com maior indicação de auxílio, o de vestir-se.

Na avaliação da qualidade de vida relacionada à saúde, por meio de instrumento genérico, o índice físico (CHQ) mostrou diferença significante entre os dois grupos, com os maiores índices no grupo oligoarticular, indicando melhor estado de saúde, mas os valores do índice psicossocial (CHQ), embora fossem maiores na forma oligoarticular, não diferiram estatisticamente da forma poliarticular. Esses resultados, em conjunto, sinalizam diferenças na percepção sobre o estado de saúde em relação à avaliação subjetiva pelo médico e pelas medidas clínicas da atividade.

Verificou-se diferença significante nos índices capacidade funcional (CHAQ), físico e psicossocial (CHQ), após mudanças efetivas no curso de tratamento, observadas, na segunda visita, em 13 pacientes do grupo poliarticular. A análise da responsividade, a medida estatística para avaliar as mudanças na capacidade funcional e do estado de saúde, é apresentada na Tabela 3. As medidas objetivas, que incluem o número de articulação ativas, com limitação dos movimentos, e a velocidade de hemossedimentação apre-

Tabela 1 - Caracterização clínica e classificação dos pacientes com artrites idiopáticas juvenis

\begin{tabular}{lc}
\hline Variável & $\mathbf{N}^{\mathbf{0}}$ \\
\hline Subtipo de apresentação & \\
$\quad$ Oligoarticular & 22 \\
$\quad$ Poliarticular & 10 \\
$\quad$ Sistêmico & 4 \\
Subtipo da evolução & \\
$\quad$ Oligoarticular & 17 \\
Poliarticular & 19 \\
Atividade da doença & \\
$\quad$ Ativa & 20 \\
Estável & 10 \\
Inativa & 3 \\
Em remissão & 3 \\
\hline
\end{tabular}


Tabela 2 - Comparação entre as medidas da atividade da doença em 36 pacientes com artrites idiopáticas juvenis

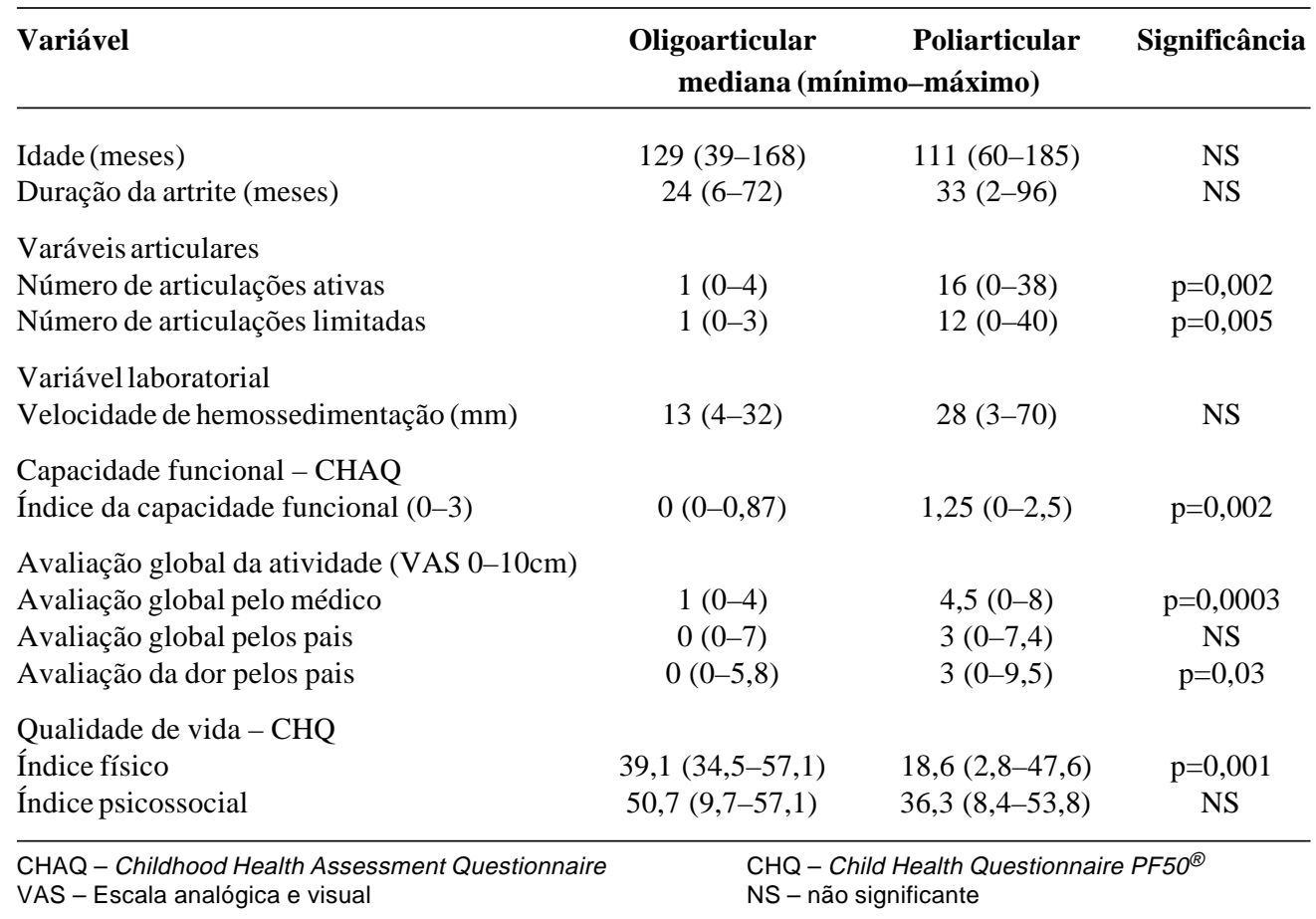

sentaram maior responsividade. As medidas avaliadas por meio de instrumentos auto-aplicáveis, os índices de capacidade física (CHAQ), índice físico e psicossocial (CHQ) apresentaram responsividade intermediária. Entre a medi- das subjetivas, a avaliação global da atividade pelo médico foi a mais responsiva, tendo os índices subjetivos de avaliação da dor e bem-estar global pelos pais menor responsividade.

Tabela 3 - Análise estatística da responsividade das medidas de atividade no grupo com artrite idiopática juvenil poliarticular avaliada em duas visitas consecutivas

\begin{tabular}{|c|c|c|c|c|}
\hline Variável & $\begin{array}{c}\text { Mediana } \\
\text { Valores basais }\end{array}$ & $\begin{array}{l}\text { Diferença } \\
\text { Medianas }\end{array}$ & SRM & ES \\
\hline \multicolumn{5}{|l|}{ Avaliação global da atividade (VAS 0-10cm) } \\
\hline Avaliação global pelo médico & 5 & 2,5 & 5,5 & 2,4 \\
\hline Avaliação global pelos pais & 3,6 & 1,7 & 3,8 & 1,7 \\
\hline Avaliação da dor pelos pais & 3,5 & 1,75 & 2,3 & 3,9 \\
\hline \multicolumn{5}{|l|}{ Capacidade funcional (CHAQ) } \\
\hline \multicolumn{5}{|l|}{ Qualidade de vida (CHQ) } \\
\hline Índice físico & 18,6 & $-14,8$ & 4,8 & 0,7 \\
\hline Índice psicossocial & 36,3 & $-13,9$ & 2,7 & 1,3 \\
\hline \multicolumn{5}{|l|}{ Variáveis articulares } \\
\hline Número de articulações ativas & 22 & 14 & 3,7 & 2,7 \\
\hline Número de articulaçoes limitadas & 17 & 11 & 4,9 & 2,4 \\
\hline \multicolumn{5}{|l|}{ Variável laboratorial } \\
\hline Velocidade de hemossedimentação (mm) & 35 & 11,5 & 7,7 & 0,7 \\
\hline
\end{tabular}




\section{Discussão}

A versão pediátrica do Health Assessment Questionnai$r e^{11,18,19}$, o Childhood Health Assessment Questionnaire $(C H A Q)$, com adaptação cultural em diversos idiomas ${ }^{20}$, possibilita, com vantagem, o uso deste instrumento para avaliação de artrite crônica desde a infância até a vida adulta. Confirmamos, no presente estudo, a sua aplicabilidade, avaliando o perfil da capacidade funcional de acordo com a percepção dos pais nas consultas de rotina. $O$ índice da capacidade funcional, assim como os índices das 8 categorias do $\mathrm{CHAQ}$, indicaram maior incapacidade e atividade no grupo poliarticular. O uso infreqüente de aparelhos e dispositivos especiais para a realização de atividades diárias pode refletir uma característica sociocultural quanto à independência nas atividades, pois o auxilio de outra pessoa em diversas categorias de atividades foi registrada em grande proporção de casos do grupo poliarticular. O grupo oligoarticular apresentou menor repercussão funcional, contudo, a avaliação do bem-estar global, sob a percepção dos pais, não diferiu do poliarticular. Isso pode refletir diferenças na avaliação subjetiva e percepção sobre a doença, como observado por Ruperto e col. $1999^{17}$.

O efeito completo da artrite sobre o paciente e o seu prognóstico não é inteiramente captado sem o uso de um instrumento genérico de avaliação da qualidade de vida. Tal como o Medical Outcome Health Survey (SF36) para adultos $^{21}$, o Child Health Questionnaire (CHQ) tem sido utilizado em diversas doenças crônicas pediátricas ${ }^{15}$, e as versões adaptadas para o português foram publicadas na íntegra recentemente ${ }^{13,14}$.

Há ampla variedade no prognóstico da artrite crônica em crianças ${ }^{22-26}$, e a percepção de diferentes dimensões do prognóstico pode variar em diferentes culturas e sociedades $^{5,25,26}$, contudo, há poucos estudos publicados sobre a qualidade de vida relacionada à saúde em países não industrializados. Este conceito inclui o impacto da incapacidade física causada pela doença no desempenho escolar, na vida social, e nas mais diversas atividades dentro e fora do âmbito familiar, ampliando a dimensão do impacto da doença sobre a criança e a sua família. No estudo de Feldman e col. ${ }^{7}$ foram comparados dois instrumentos genéricos, a qualidade de vida senso lato e a qualidade de vida relacionada ao estado de saúde, com um instrumento específico, o índice da capacidade física do CHAQ, e tendo encontrado concordância entre as medidas, enfatizaram a importância de se aplicar simultaneamente a avaliação global e genérica da qualidade de vida, a capacidade funcional e as medidas clínicas de atividade da doença, tanto na prática diária como em ensaios terapêuticos.

A responsividade é a medida estatística adequada para avaliar mudanças no estado de saúde, ou na percepção sobre o estado de saúde, mediante intervenção terapêutica. Para ser qualificada como responsiva, uma medida deve ter mudanças consistentes ao longo do tempo, ou ser comparada a outra medida de valor conhecido ${ }^{28}$. Em consonância com outros estudos ${ }^{17,27}$, verificamos maior responsividade da avaliação global pelo médico e menor responsividade nas medidas de percepção pelos pais, entre as medidas subjetivas, tendo as medidas avaliadas por instrumento, sensibilidade relativa para estimar a melhora clínica em pacientes com artrite e recebendo tratamento específico. A despeito da menor responsividade, concluímos que a dimensão física e psicossocial, avaliada de forma abrangente por meio de instrumentos, é aplicável e útil no atendimento clínico rotineiro de pacientes. Isso também poderá facilitar a sua aplicação prática em futuros ensaios terapêuticos para as artrites idiopáticas juvenis.

\section{Referências bibliográficas}

1. Ba'Pham, Klassen R. Assessing clinical measures and clinical disagreement. In: Moyer, VA Elliott EJ, Davis RL, Gilbert R, Klassen T, Logan S, et al., editores. Evidence-based Pediatrics and Child Health. London: BMJ Books - BMJ Publishing Group; 2000.p.71-78.

2. Waters E. Assessing quality of life. In: Moyer VA, Elliott EJ, Davis RL, Gilbert R, Klassen T, Logan S, et al., editores. Evidence-based Pediatrics and Child Health. London: BMJ Books - BMJ Publishing Group; 2000.p.79-90.

3. Jenney MEM, Campbell S. Measuring quality of life. Arch Dis Child 1997;77:347-54.

4. Strand CV, Russel AS. WHO/ILAR task force on quality of life. J Rheumatol 1997;24:1630-3.

5. Burgos-Vargas R. Assessment of quality of life in children with rheumatic disease [editorial]. J Rheumatol 1999;26:1432-4.

6. Tucker LB. Whose life is it anyway? Understanding quality of life in children with rheumatic diseases [editorial]. J Rheumatol 2000;27:8-11.

7. Feldman BM, Grundland B, McCullough L, Wright V. Distinction of quality of life, health related quality of life and health status in children referred for rheumatologic care. J Rheumatol 2000;27:226-33.

8. Petty RE, Southwood TR, Baum J, Bhettay E, Glass DN, Manners P, et al. Revision of the proposed classification criteria for juvenile idiopathic arthritis. Durban 1997. J Rheumatol 1998;25:1991-4.

9. Arguedas OA. Juvenile chronic arthritis - A study on epidemiology and outcome in Costa Rican children [dissertação]. Göteborg University-Sweden; 2001.

10. Giannini EH, Ruperto N, Ravelli A, Lovell DJ, Martini A. For the Pediatric Rheumatology International Trials Organization. Preliminary definition of improvement in juvenile arthritis. Arthritis Rheum 1997;40:1202-9.

11. Singh G, Athreya BH, Fries JF, Goldsmith DP. Measurement of health status in children with juvenile rheumatoid arthritis. Arthritis Rheum 1994;37(12):1761-9.

12. Len C, Goldenberg J, Ferraz MB, Hilario MO, Oliveira LM, Sacchetti S. Cross cultural reliability of the Childhood Health Assessment Questionnaire. J Rheumatol 1994;21:2349-52.

13. Melo-Gomes JA, Ruperto N, Canhão H, Fonseca JA, Quintal A, Salgado M, et al. for the Pediatric Rheumatology International Trials Organisation (PRINTO) The Portuguese version of the Childhood Health Assessment Questionnaire (CHAQ) and the Child Health Questionnaire (CHQ). Clin Exp Rheumatol 2001;19 (Suppl 23):126-130. 
14. Machado CSM, Ruperto N, Silva CHM, Ferriani VPL, Roscoe I, Campos LMA, et al. for the Pediatric Rheumatology International Trials Organisation (PRINTO) The Brazilian version of the Childhood Health Assessment Questionnaire (CHAQ) and the Child Health Questionnaire (CHQ). Clin Exp Rheumatol 2001;19 (Suppl 23):25-29.

15. Landgraf JM, Albertz L, Ware JE Jr. The Child Health Questionnaire: A user's manual. $2^{\mathrm{a}}$ ed. Boston, The Health Act; 1999.

16. Guillemin F, Bombardier C, Beaton D. Cross-cultural adaptation of health-related quality of life measures: literature review and proposed guidelines. J Clin Epidemiol 1993;46:1417-32.

17. Ruperto N, Ravelli A, Migliavacca D, Viola S, Pistorio A, Duarte $\mathrm{C}$, et al. Responsiveness of clinical measures in children with oligoarticular juvenile chronic arthritis. J Rheumatol 1999;26:1827-30.

18. Fries JF, Spitz P, Kraines RG, Holman HR Measurement of patient outcome in arthritis. Arthritis Rheum 1980;23:137-45.

19. Timko C, Stovel KW, Moos RH, Miller JJ III Adaptation to juvenile rheumatic diseases. A controlled evaluation of functional disability with a one-year follow up. Health Psychology 1992;11:67-76.

20. Ruperto N, Ravelli A, Pistorio A, Mallattia C, Cavuto S, GadoWest L, et al. For the Pediatric Rheumatology International Trials Organisation (PRINTO) Cross-cultural adaptation and psychometric evaluation of the Childhood Health Assessment Questionnaire (CHAQ) and the Child Health Questionnaire (CHQ) in 32 countries. Review of the general methodology. Clin Exp Rheumatol 2001;19 (Suppl 23):1-9.

21. Ware JE Jr, Sherbourne CD. The MOS 33-item short-form health survey (SF-36) Conceptual framework and item selection. Med Care 1992;30:473-83.

22. Wallace CA, Levinson JE. Juvenile rheumatoid arthritis: outcome and treatment for the 1990s. Rheum Dis Clin North Am 1991;17:891-905.
23. Andersson Gare B, Fasth A. The natural history of juvenile chronic arthritis: a population based cohort study I. Onset and disease process. J Rheumatol 1995;22:295-307.

24. Andersson-Gäre B. Juvenile Arthritis - Who gets it, where and when? A review of current data in incidence and prevalence. Clin Exp Rheumatol 1999;17:367-74.

25. Ruperto N, Levinson JE, Ravelli A, Shear ES, Tague L, Murray $\mathrm{K}$, et al. Long term health outcomes and quality of life in American and Italian inception cohorts of patients with juvenile rheumatoid arthritis. I. Outcome Status. J Rheumatol 1997;24:945-51.

26. Ruperto N, Levinson JE, Ravelli A, Shear ES, Tague L, Murray $\mathrm{K}$, et al. Long term Health Outcomes and Quality of Life in American and Italian Inception Cohorts of Patients with Juvenile Rheumatoid Arthritis. II. Early predictors of outcome. J Rheumatol 1997;24:952-8.

27. Ruperto N, Ravelli A, Falcini F, Lepore L, Buoncompagni A, Gerlone V, et al. Responsiveness of outcome measures in juvenile chronic arthritis. Rheumatology 1999;38:176-80.

28. Dempster H, Porepa M, Young N, Feldman BM. The clinical meaning of functional outcome scores in children with Juvenile Arthritis. Arthritis Rheum 2001;44:1766-74.

\section{Endereço para correspondência:}

Dra. Claudia S.M. Machado

Departamento de Pediatria - Faculdade de Medicina de Botucatu, UNESP

CEP 18618-970 - Botucatu, SP

Fone/Fax: (14) 6802.6274 / 6802. 6083 / 6822.0421

E-mail:cmachado@fmb.unesp.br 